\section{Microbiological characteristics of nosocomial pneumonia in surgical patients: one size doesn't fit all.}

\author{
A. Benítez-Cano, S. Bermejo, L. Castelltort, L. Bosch, C. García-Bernedo, J. Vallés \\ Hospital del Mar, Dept. of Anaesthesiology \& Intensive Care, Barcelona, Spain.
}

\section{BACKGROUND AND GOAL OF THE STUDY}

Postoperative pneumonia (POP) is associated with an increase on morbidity, mortality and length of hospital stay. Multi-drug resistant pathogens (MDR) could be present in respiratory samples in POP early after hospital admission (48h) even in patients without risk factors.

The aim of the study is to assess microbiological features of POP in a surgical intensive care unit (SICU) and determine if risk factors for MDR germs (RFMDR) are still able to predict their isolation.

\section{MATERIAL AND METHODS}

We retrospectively analysed data from POP diagnosed during the admission in the SICU (January/10-November/17).

Variables analysed: demographic, microbiological respiratory cultures and RFMDR: $\geq 5$ days of hospitalization prior to the occurrence of POP, antimicrobial therapy in the preceding 90 days, hospitalization for $\geq 2$ days in the preceding 90 days.

\section{RESULTS AND DISCUSSION}

- 195 patients with an average age of 70 (18-93) years old had at least one episode of POP, 83\% ASA III-IV and 95\% were intubated in the operating room.

- A total of 267 episodes of POP were recorded. Pathogens were isolated in $70 \%$ of cases (206 isolations).

- In the group of patients with FRMDR, MDR pathogens were isolated in $31 \%$ of episodes (Figure 1 ). In $92 \%$ of cases, MDR isolation could be predicted by RFMDR.

- Most sensitive pathogens were isolated on early SICU admission in patients without RFMDR (Figure 2 and Figure 3). P. aeruginosa and Haemophilus spp. were the most frequent germs in this group (24\%).

- S.pneumoniae S.aureus P.aeruginosa Klebsiella spp.

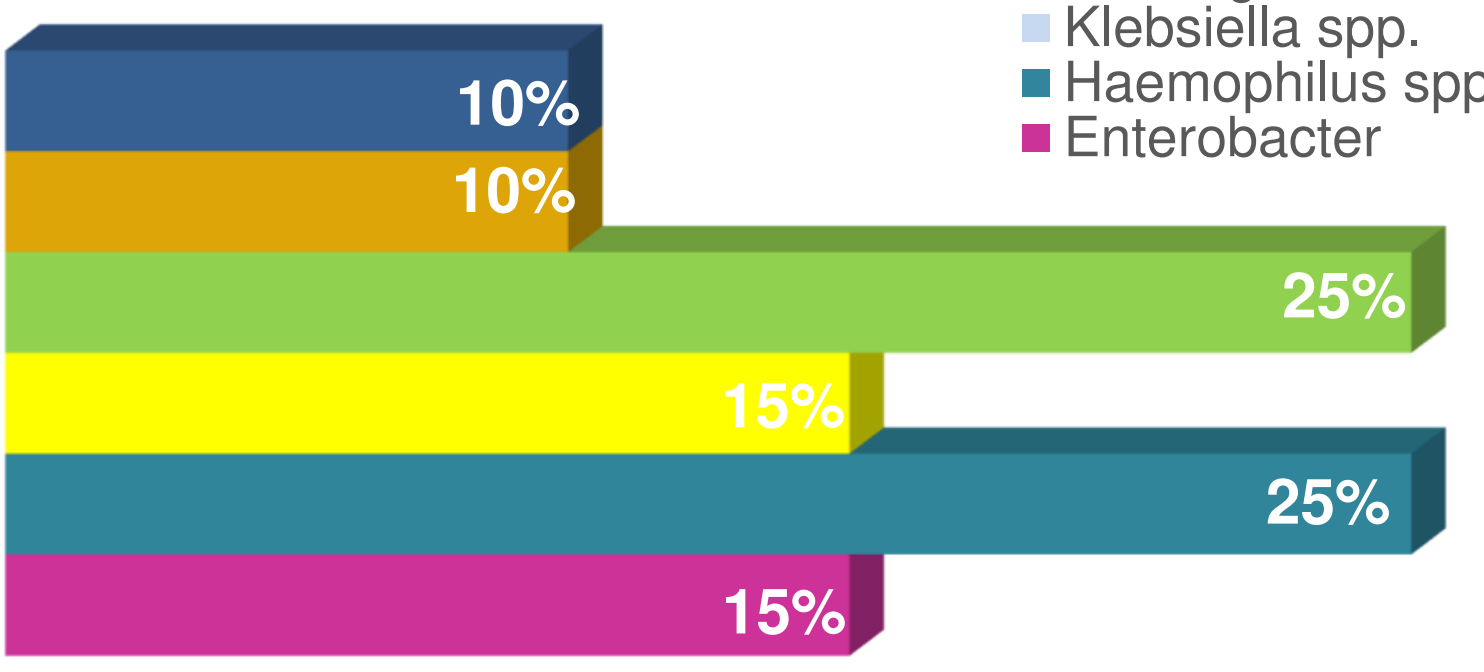

Figure 3. Distribution of sensitive bacteria in early $P O P$ in patients without RFMDR

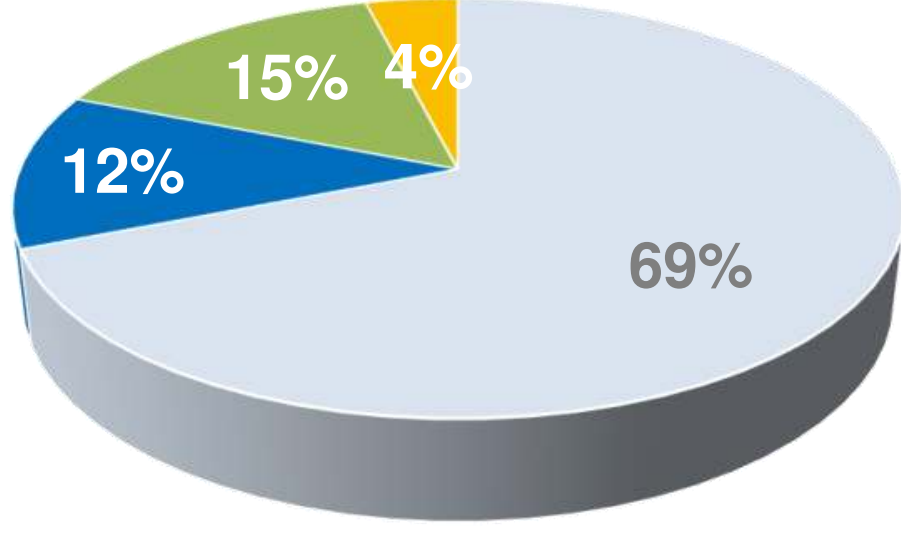

Figure 1. Microbiology of POP in patients with RFMDR

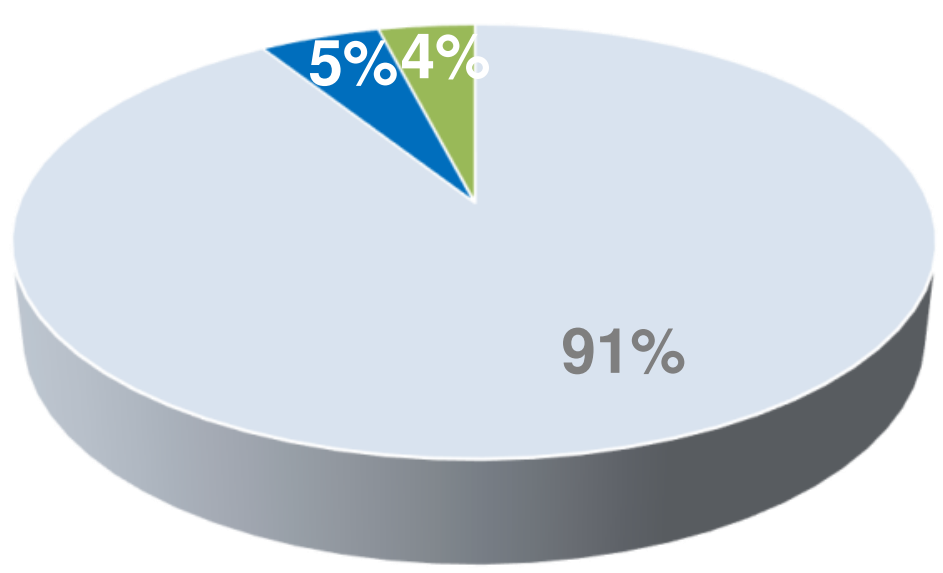

Figure 2. Microbiology of $P O P$ in patients without RFMDR

\section{CONCLUSIONS}

On a tertiary SICU, no matter time on admission, MDR pathogens isolation are still predictable by RFMDR. Taking into account our local flora, empiric coverage of early POP in patients without RFMDR should include Ps. Aeruginosa and Gram-negative bacteria (Enterobacter, Klebsiella spp.), that are not commonly isolated in community pneumonia.

\section{REFERENCES}

1. Montravers P et al. Crit Care Med 2002.

2. Kalil AC et al. Clin Infect Dis 2016. 